\title{
Sleep quality and mental health of medical workers during the coronavirus disease 2019 pandemic
}

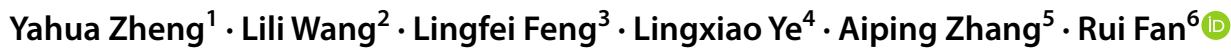

Received: 2 September 2020 / Accepted: 24 December 2020 / Published online: 10 January 2021

(C) Japanese Society of Sleep Research 2021

\begin{abstract}
The purpose of this study is to assess the sleep quality, mental health status, and associated factors among medical workers during the coronavirus disease 2019 (COVID-19) pandemic. A cross-sectional study was conducted and medical workers in Ningbo, China were recruited. Sleep quality was evaluated by Pittsburgh Sleep Quality Index (PSQI). Mental health status was evaluated by Symptom Checklist 90 (SCL-90). Logistic regression and generalized multi-factor dimensionality reduction (GMDR) analysis were utilized to explore the risk factors and their interactions on sleep quality and mental health status. 207 participants were surveyed, and 34.30\% were found with poor sleep quality (total PSQI score > 10), mainly manifested as sleep disturbance (92.75\%). 27.05\% were found with mental symptoms (Global severity index $>1.50$ ), mainly manifested as obsessive-compulsive (25.60\%). Multivariate logistic analysis showed that male (OR 3.89, 95\% CI 1.06-14.24, $P=0.04$ ), working years $>15$ years (OR 4.51, 95\% CI 1.56-13.00, $P=0.01$ ), nurse (OR 5.64, 95\% CI 1.35-23.63, $P=0.02$ ), more night shifts (OR 3.10, 95\% CI 1.31-7.34, $P=0.01$ ), and supporting Wuhan (OR 3.41, 95\% CI 1.12-10.40, $P=0.03$ ) were associated with poor sleep quality. GMDR analysis showed that there was a two-factor interaction between working years and working shifts $(P=0.01)$. No significant factors and interactions were found associated with mental symptoms. In conclusions, about one-third of medical workers suffered from sleep and mental problems during the COVID-19 pandemic in the current study. Interventions for sleep and mental problems among medical workers were needed based on related factors.
\end{abstract}

Keywords Sleep quality $\cdot$ Mental health $\cdot$ Medical workers $\cdot$ COVID-19

Yahua Zheng and Lili Wang have contributed equally to this work.

Rui Fan

frnbdx@126.com

1 Nursing Department, Ningbo Medical Center Lihuili Hospital, Ningbo 315040, Zhejiang, China

2 Neurosurgery Department, Ningbo Medical Center Lihuili Hospital, Ningbo 315040, Zhejiang, China

3 Pediatrics Department, Cixi Maternity and Child Health Care Hospital, Ningbo 315300, Zhejiang, China

4 Cardiology Department, Ningbo Medical Center Lihuili Hospital, Ningbo 315040, Zhejiang, China

5 Department of Chronic Disease Control and Prevention, Zhenhai Center for Disease Control and Prevention, Ningbo 315200, Zhejiang, China

6 Medical Quality Management Office, Ningbo Medical Center Lihuili Hospital, Ningbo 315040, Zhejiang, China

\section{Introduction}

Coronavirus disease 2019 (COVID-19), formerly known as 2019 novel coronavirus (2019-nCoV) and severe acute respiratory syndrome coronavirus 2 (SARS-CoV-2), has spread internationally. According to data published by the World Health Organization, a total of 25,541,380 cases were confirmed worldwide, 852,000 patients died as of September 2, 2020 [1].

The COVID-19 pandemic not only caused great public concern, but also led to huge mental burden and sleep disturbances, especially for medical workers [2-8]. Previous studies have indicated adverse mental reactions to the 2003 SARS outbreak among health care workers $[9,10]$. Recently, studies showed that more than one-third of the medical workers suffered from insomnia symptoms [2], $26.67 \%$ of front-line medical workers suffered from severe insomnia [3], 60\% suffered from insomnia combined with moderate-severe stress [11] during the COVID-19 pandemic. However, the prevalence rates of sleep and mental 
problems were significantly different among various studies and groups.

In addition, sleep quality and mental health of medical workers were associated with lots of factors during the COVID-19 pandemic. Chenxi Zhang et al. showed that occupation, education level, an isolation environment, and psychological worry about the COVID-19 pandemic were related to insomnia [2]. Those in Wuhan, nurses, women, and front-line medical workers suffered from psychological problems [6]. Professional background and female were the predictors of poor sleep quality combined with moderate-severe stress [11]. Although several insomnia-related and mental-related factors have been reported, there was no consistent conclusion on the type of factors, the size of factor effect. Furthermore, sleep and mental problems are complex multifactorial problems, the interactions of multiple factors should be concerned, but there was little research on this aspect. Therefore, we aimed to assess the sleep quality and mental health status, and explore the related factors among medical workers during the COVID-19 pandemic.

\section{Materials and methods}

\section{Study participants}

A cross-sectional, survey-based study was performed among medical workers from multiple hospitals in Ningbo City, Zhejiang province, China, including medical workers who supported Wuhan. Data were collected with a self-rated, anonymous questionnaire by Wenjuanxing (www.wjx.cn) which was delivered through the internet from 1st March 2020 to 15th March 2020. Wenjuanxing is a professional online questionnaire survey platform in China, focusing on providing users with powerful and humanized online questionnaire design, data collection, and data analysis services. The people with valid telephone number can register. All subjects provided informed consent electronically prior to survey. Only subjects who chose yes on the informed consent page were surveyed, and subjects could quit the process at any time. This study was approved by the ethics committee of Ningbo Medical Center Lihuili Hospital (KY2020PJ066).

\section{Questionnaire}

The questionnaire consisted of three parts: basic demographic information, sleep quality assessment (the Pittsburgh Sleep Quality Index), and mental health assessment (the Symptom Checklist 90).

Basic demographic information included age $(\leq 30$ years or $>30$ years), gender (male or female), working years (1-5 years, $6-10$ years, $11-15$ years, $>15$ years), educational level (college, undergraduate, postgraduate), occupation (doctor, nurse, and technician), type of hospital (Grade I hospital or community, Grade II hospital, Grade III hospital), working shifts (as usual, more night shifts, and more day shifts), working position (front line or second line), and whether to support Wuhan (yes or no). Grade I hospital or community is the primary health care institution that directly provided comprehensive services of treatment, prevention, and rehabilitation for the community. Grade II hospital is the regional hospital that provided medical services across several communities and is the technical center of regional medical prevention. Grade III hospital is the hospital that provided medical services across regions, cities, provinces, and the whole country. It is the medical prevention technology center with comprehensive medical, teaching, and scientific research capabilities. Medical workers who were directly engaged in clinical activities of diagnosing, treating, or providing nursing care to COVID-19 confirmed or suspected patients were defined as frontline workers.

\section{Sleep quality assessment}

Sleep quality was evaluated with the Pittsburgh Sleep Quality Index (PSQI) [12, 13]. The PSQI includes 7 components and 18 entries in total. Each entry is scored with a 0-3 scale. The 7 components include subjective sleep quality (positive: 2-3 score, negative: $0-1$ score), time to sleep (positive: $2-3$ score, negative: $0-1$ score), sleep time (positive: $2-3$ score, negative: $0-1$ score), sleep efficiency (positive: $2-3$ score, negative: $0-1$ score), sleep disturbance (positive: $1-3$ score, negative: 0 score), hypnotic drugs (positive: $1-3$ score, negative: 0 score), and daytime dysfunction (positive: $1-3$ score, negative: 0 score). Of them, the positive for subjective sleep quality indicates subjective sleep quality is poor, the positive for time to sleep indicates time to sleep is long, the positive for sleep time indicates sleep time is $<6 \mathrm{~h}$, and the positive for sleep efficiency indicates sleep efficiency is poor. The cumulative score of each component is the total PSQI score varying from 0 to 21 . The higher scores the people get, the worse the sleep quality is. $0-5$ scores indicate good sleep quality, 6-10 scores indicate average sleep quality, 11-15 scores indicate poor sleep quality, and 16-21 scores indicate very poor sleep quality. Thus, a total score $>10$ was identified as poor sleep quality in the current study. The PSQI has been widely used in previous studies with high reliability and validity, and the Cronbach's alpha was 0.811 [14].

\section{Mental health assessment}

Mental health was evaluated with the Symptom Checklist 90 (SCL-90) [15, 16]. There are 90 items in total. 90 items made up 10 factors. Each item is scored with a 5-point Likert scale, ranging from 0 (none) to 4 (severe). Items are calculated and converted to get the total score and subscale scores. 
The 10 factors include somatization (positive: $\geq 24$ score, negative: $<24$ score), obsessive-compulsive (positive: $\geq 20$ score, negative: $<20$ score), interpersonal sensitivity (positive: $\geq 18$ score, negative $<18$ score), depression (positive: $\geq 26$ score, negative: $<26$ score), anxiety (positive: $\geq 20$ score, negative: $<20$ score), hostility (positive: $\geq 12$ score, negative: $<12$ score), photic anxiety (positive: $\geq 14$ score, negative: $<14$ score), paranoididefition (positive: $\geq 12$ score, negative: $<12$ score), psychotieism (positive: $\geq 20$ score, negative: $<20$ score), and other (positive: $\geq 14$ score, negative: $<14$ score). If any subscale score is higher than 2 , positive items are higher than 43 , or the total score is higher than 160, it suggests psychological abnormality. The Global Severity Index (GSI) ranging from 1 to 5 is calculated as the mean of all 90 items, which is considered the overall index of mental symptoms. The higher scores the people get, the worse the mental health is. 1-1.5 scores indicate none mental symptom, 1.5-2.5 scores indicate mild mental symptom, 2.5-3.5 scores indicate moderate mental symptom, 3.5-4.5 scores indicate moderate-to-severe mental symptom, and 4.5-5 points indicate severe mental symptom. Thus, GSI $>1.5$ is defined with mental symptom in the current study. The SCL-90 has been widely used in the previous studies with high reliability and validity, and the Cronbach's alpha was 0.983 [17].

\section{Statistical analysis}

The original scores of above two measurement tools were not normally distributed and so were presented as medians with interquartile ranges (IQRs). Categorical variables were presented as percentages and analyzed using the Chi-square test. To explore the associations among demographic factors and sleep quality, and mental health, logistic regression was used. In addition, the generalized multi-factor dimensionality reduction (GMDR) method was used to explore potential high-order interactions [18, 19]. Due to the influence of "dimension disaster", traditional statistical models were not suitable for exploring potential high-order interactions. As a non-parametric testing method, GMDR could overcome the influence of "dimension disaster" and correct the confounding factors, which significantly improved the accuracy of prediction. Through GMDR method, high-dimensional data were finally transformed into one-dimensional data with two levels ("high risk", "low risk"), and the confounding factors were adjusted. In our analysis, the data were randomly divided into 10 equal parts, 9 of which were used as training samples for the construction of the interaction model, and the remaining one was used as a test sample for the test of the model. According to the analysis results, the model with $P$ value less than 0.05 and the largest crossvalidation consistency and maximum prediction accuracy was selected as the best model. A $P$ value of $<0.05$ was considered statistically significant. Data analysis was performed using SPSS statistical software version 20.0 (IBM Corp) and GMDR v0.7 program (http://ibi.zju.edu.cn/softw are/GMDR/download.html).

\section{Results}

\section{Characteristics of the study population}

A total of 207 participants were surveyed in our study, 131 $(63.29 \%)$ aged $>30$ years, $175(84.54 \%)$ were females, 38 $(18.36 \%)$ were doctors, $155(74.88 \%)$ were nurses, and $14(6.76 \%)$ were technicians. Additionally, most of them came from Grade III hospital [167 (80.68\%)], had worked for 6-10 years [85 (41.06\%)], and had an educational level of undergraduate [166 (80.19\%)]. During the survey, 132 $(63.77 \%)$ subjects were in the front line, 101 (48.79\%) were supporting Wuhan, and 87 (42.03\%) mainly worked in the day shift (Table 1).

\section{Assessment of sleep quality and associated factors}

As a result, the average total PSQI score was 9 in the 207 studied subjects, and $71(34.30 \%)$ subjects had the total PSQI score $>10$ and defined as poor sleep quality. 91 $(43.96 \%)$ were positive for subjective sleep quality, 125 $(60.39 \%)$ were positive for time to sleep, 70 (33.82\%) were positive for sleep time, $98(47.34 \%)$ were positive for sleep efficiency, $192(92.75 \%)$ were positive for sleep disturbance, $49(23.67 \%)$ were positive for hypnotic drugs, and 162 (78.26\%) were positive for daytime dysfunction.

Univariate logistic analysis showed that working years (11-15 years) (OR 5.28, 95\% CI 2.03-13.76, $P=1.00 \times 10^{-3}$ ), working shifts (more night shifts) (OR 2.94, 95\% CI 1.37-6.31, $P=0.01$ ), working position (front line) (OR 1.92, 95\% CI 1.02-3.59, $P=0.04$ ), and whether to support Wuhan (yes) (OR 2.71, 95\% CI 1.49-4.92, $P=1.00 \times 10^{-3}$ ) were associated with poor sleep quality. After adjusting for confounding factors, multivariate logistic analysis showed that gender (male) (OR 3.89, 95\% CI 1.06-14.24, $P=0.04$ ), working years ( $>15$ years) (OR 4.51, 95\% CI 1.56-13.00, $P=0.01$ ), occupation (nurse) (OR 5.64, 95\% CI 1.35-23.63, $P=0.02$ ), working shifts (more night shifts) (OR 3.10, 95\% CI 1.31-7.34, $P=0.01$ ), and supporting Wuhan (yes) (OR 3.41, 95\% CI 1.12-10.40, $P=0.03$ ) were associated with poor sleep quality (Table 2 ).

Furthermore, GMDR interaction analysis of sleep quality was conducted among those factors which were significant by multivariate logistic regression analysis, including gender, working years, occupation, working shifts, and supporting Wuhan. According to the screening principle of the best model (with a sign test $P$ value of $<0.05$, and the highest 
Table 1 Characteristics of the study population $(n=207)$

\begin{tabular}{|c|c|c|}
\hline Variables & $\begin{array}{l}\text { Number } \\
(n=207)\end{array}$ & Percentage \\
\hline \multicolumn{3}{|l|}{ Age (years) } \\
\hline$\leq 30$ & 76 & 36.71 \\
\hline$>30$ & 131 & 63.29 \\
\hline \multicolumn{3}{|l|}{ Gender } \\
\hline Male & 32 & 15.46 \\
\hline Female & 175 & 84.54 \\
\hline \multicolumn{3}{|l|}{ Working years (years) } \\
\hline $1-5$ & 43 & 20.77 \\
\hline $6-10$ & 85 & 41.06 \\
\hline $11-15$ & 39 & 18.84 \\
\hline$>15$ & 40 & 19.32 \\
\hline \multicolumn{3}{|l|}{ Educational level } \\
\hline College & 29 & 14.01 \\
\hline Undergraduate & 166 & 80.19 \\
\hline Postgraduate & 12 & 5.80 \\
\hline \multicolumn{3}{|l|}{ Occupation } \\
\hline Doctor & 38 & 18.36 \\
\hline Nurse & 155 & 74.88 \\
\hline Technician & 14 & 6.76 \\
\hline \multicolumn{3}{|l|}{ Type of hospital } \\
\hline Grade I hospital or community & 8 & 3.86 \\
\hline Grade II hospital & 32 & 15.46 \\
\hline Grade III hospital & 167 & 80.68 \\
\hline \multicolumn{3}{|l|}{ Working shifts } \\
\hline As usual & 60 & 28.99 \\
\hline More night shifts & 60 & 28.99 \\
\hline More day shifts & 87 & 42.03 \\
\hline \multicolumn{3}{|l|}{ Working position } \\
\hline Frontline & 132 & 63.77 \\
\hline Second line & 75 & 36.23 \\
\hline \multicolumn{3}{|l|}{ Supporting Wuhan } \\
\hline Yes & 101 & 48.79 \\
\hline No & 106 & 51.21 \\
\hline Total PSQI score, $M$ (IQR) & - & $9.00(7.00)$ \\
\hline \multicolumn{3}{|l|}{ Sleep quality } \\
\hline Poor (total PSQI score > 10) & 71 & 34.30 \\
\hline Good (total PSQI score $\leq 10$ ) & 136 & 65.70 \\
\hline Total SCL-90 score, $M$ (IQR) & - & $106.00(47.00)$ \\
\hline GSI, $M$ (IQR) & - & $1.18(0.52)$ \\
\hline \multicolumn{3}{|l|}{ Mental symptom } \\
\hline Positive (GSI > 1.5) & 56 & 27.05 \\
\hline Negative (GSI $\leq 1.5$ ) & 151 & 72.95 \\
\hline
\end{tabular}

$P S Q I$ Pittsburgh sleep quality index, GSI global severity index, IQR interquartile range

cross-validation consistency, prediction accuracy), the twofactor interaction model of working years with working shifts was selected (Table 3).
Assessment of mental health and associated factors

As shown in Table 4, the total SCL score was 106, global severity index (GSI) was 1.18 in the studied population, and $56(27.05 \%)$ subjects had mental symptom (GSI > 1.5). 26 (12.56\%) were positive for somatization, $53(25.60 \%)$ were positive for obsessive-compulsive, 34 (16.43\%) were positive for interpersonal sensitivity, $30(14.49 \%)$ were positive for depression, $30(14.49 \%)$ were positive for anxiety, 31 (14.98\%) were positive for hostility, 20 (9.66\%) were positive for photic anxiety, 25 (12.08\%) were positive for paranoididefition, 25 (12.08\%) were positive for psychotieism, and $56(27.05 \%)$ were positive for other. However, no significant factors were found associated with mental symptom by logistic analysis (Table 5), and no interactions were found by GMDR analysis (data not shown).

\section{Discussion}

Our research found that about one-third of medical workers suffered from sleep problems (mainly manifested as sleep disturbance) and mental problems (mainly manifested as obsessive-compulsive). Male, working years $>15$ years, nurse, more night shifts, supporting Wuhan, and a two-factor interaction between working years and working shifts were all risk factors for sleep quality. Interventions for sleep and mental problems among medical workers were needed.

Previous studies indicated that the insomnia rate was $34.2-37 \%$ during the SARS pandemic $[20,21]$ and $36.1 \%$ among medical staff during the COVID-19 pandemic [2]. Similarly, the present study found that the prevalence rate of poor sleep quality was $34.30 \%$, and the average PSQI score of medical workers was 9 , which meant that the overall sleep quality of medical workers during the COVID-19 pandemic was poor. The poor sleep quality might be due to the overload work and intense psychological pressure of medical workers. Of note, Haitham Jahram et al. [11] found that $75-76 \%$ healthcare workers were poor sleepers, which was higher than ours. The difference might be related to the different populations and different definitions of poor sleep quality. In a word, medical institutions should improve infectious disease prevention and control system, strengthen psychological counseling and humanistic care for medical workers, and improve their sleep quality and mental health status.

The current study demonstrated that male, working years $>15$ years, nurse, more night shifts, and supporting Wuhan were risk factors for poor sleep quality. Similarly, previous studies found that nurses were more susceptible to insomnia [2]. The reasons were as follows: in clinical work, doctors often work in the daytime, while nurses may have to work the whole night with frequent night shifts [22], 
Table 2 Logistic regression analysis of risk factors associated with sleep quality

\begin{tabular}{|c|c|c|c|c|c|}
\hline \multirow[t]{2}{*}{ Variables } & \multirow{2}{*}{$\begin{array}{l}\text { No. of cases/no. of total } \\
\text { cases }(\%)\end{array}$} & \multicolumn{2}{|l|}{ Model 1} & \multicolumn{2}{|l|}{ Model 2} \\
\hline & & OR $(95 \%$ CI $)$ & $P$ & OR $(95 \%$ CI $)$ & $P$ \\
\hline \multicolumn{6}{|l|}{ Age (years) } \\
\hline$\leq 30$ & $21 / 76(27.63)$ & 1.00 (reference) & NA & 1.00 (reference) & NA \\
\hline$>30$ & $50 / 131(38.17)$ & $1.62(0.88-2.99)$ & 0.13 & $1.67(0.59-4.73)$ & 0.33 \\
\hline \multicolumn{6}{|l|}{ Gender } \\
\hline Male & $12 / 32(37.50)$ & $1.18(0.54-2.58)$ & 0.68 & $3.89(1.06-14.24)$ & 0.04 \\
\hline Female & $59 / 175(33.71)$ & 1.00 (reference) & NA & 1.00 (reference) & NA \\
\hline \multicolumn{6}{|l|}{ Working years (years) } \\
\hline $1-5$ & $10 / 43(23.26)$ & 1.00 (reference) & NA & 1.00 (Reference) & NA \\
\hline $6-10$ & 27/85 (31.76) & $1.54(0.66-3.57)$ & 0.32 & $0.99(0.22-4.44)$ & 0.99 \\
\hline $11-15$ & $24 / 39(61.54)$ & $5.28(2.03-13.76)$ & $1.00 \times 10^{-3}$ & $1.32(0.48-3.60)$ & 0.59 \\
\hline$>15$ & $10 / 40(25.00)$ & $1.10(0.40-3.01)$ & 0.85 & $4.51(1.56-13.00)$ & 0.01 \\
\hline \multicolumn{6}{|l|}{ Educational level } \\
\hline College & $11 / 29(37.93)$ & 1.00 (reference) & NA & 1.00 (reference) & NA \\
\hline Undergraduate & $54 / 166(32.53)$ & $0.79(0.35-1.79)$ & 0.57 & $0.48(0.18-1.27)$ & 0.14 \\
\hline Postgraduate & $6 / 12(50.00)$ & $1.64(0.42-6.36)$ & 0.48 & $2.52(0.37-17.43)$ & 0.35 \\
\hline \multicolumn{6}{|l|}{ Occupation } \\
\hline Doctor & $11 / 38(28.95)$ & 1.00 (reference) & NA & 1.00 (reference) & NA \\
\hline Nurse & $59 / 155$ (38.06) & $1.51(0.70-3.27)$ & 0.30 & $5.64(1.35-23.63)$ & 0.02 \\
\hline Technician & $1 / 14(7.14)$ & $0.19(0.02-1.62)$ & 0.13 & $0.49(0.04-5.62)$ & 0.57 \\
\hline \multicolumn{6}{|l|}{ Type of hospital } \\
\hline Grade I hospital or community & $2 / 8(25.00)$ & 1.00 (reference) & NA & 1.00 (reference) & NA \\
\hline Grade II hospital & $11 / 32(34.38)$ & $0.63(0.12-3.20)$ & 0.57 & $2.13(0.29-15.56)$ & 0.46 \\
\hline Grade III hospital & $58 / 167(34.73)$ & $0.98(044-2.18)$ & 0.97 & $1.34(0.22-8.22)$ & 0.75 \\
\hline \multicolumn{6}{|l|}{ Working shifts } \\
\hline As usual & $16 / 60(26.67)$ & 1.00 (reference) & NA & 1.00 (reference) & NA \\
\hline More night shifts & $31 / 60(51.67)$ & $2.94(1.37-6.31)$ & 0.01 & $3.10(1.31-7.34)$ & 0.01 \\
\hline More day shifts & 24/87 (27.59) & $1.05(0.50-2.20)$ & 0.90 & $1.34(0.58-3.09)$ & 0.50 \\
\hline \multicolumn{6}{|l|}{ Working position } \\
\hline Frontline & $52 / 132(39.39)$ & $1.92(1.02-3.59)$ & 0.04 & $0.34(0.10-1.15)$ & 0.08 \\
\hline Second line & $19 / 75(25.33)$ & 1.00 (reference) & NA & 1.00 (reference) & NA \\
\hline \multicolumn{6}{|l|}{ Supporting Wuhan } \\
\hline Yes & $46 / 101(45.54)$ & $2.71(1.49-4.92)$ & $1.00 \times 10^{-3}$ & $3.41(1.12-10.40)$ & 0.03 \\
\hline No & 25/106 (23.58) & 1.00 (reference) & NA & 1.00 (reference) & NA \\
\hline
\end{tabular}

Model 1: univariate logistic analysis; model 2: multivariate logistic analysis

Table 3 GMDR models of sleep quality

\begin{tabular}{llll}
\hline Model & $\begin{array}{l}\text { Prediction accu- } \\
\text { racy }\end{array}$ & Sign test $(P)$ & $\begin{array}{l}\text { Cross-valida- } \\
\text { tion consist- } \\
\text { ency }\end{array}$ \\
\hline Working shifts & 0.55 & $5(0.62)$ & $7 / 10$ \\
Working years, working shifts & 0.65 & $9(0.01)$ & $10 / 10$ \\
Working years, occupation, working shifts & 0.55 & $7(0.17)$ & $8 / 10$ \\
$\begin{array}{l}\text { Working years, occupation, working shifts, sup- } \\
\text { porting Wuhan }\end{array}$ & 0.56 & $7(0.17)$ & $7 / 10$ \\
$\begin{array}{l}\text { Gender, working years, occupation, working } \\
\text { shifts, supporting Wuhan }\end{array}$ & 0.61 & $8(0.05)$ & $10 / 10$ \\
\hline
\end{tabular}

$P$ was adjusted for age, educational level, type of hospital, and working position using logistic regression in GMDR analysis 
Table 4 Assessment of mental health status using the SCL-90

\begin{tabular}{|c|c|c|c|}
\hline \multirow[t]{2}{*}{ Variables } & \multirow[t]{2}{*}{ Total (207), $n(\%)$} & \multicolumn{2}{|c|}{ Mental symptom, $n(\%)$} \\
\hline & & Positive (56) & Negative (151) \\
\hline \multicolumn{4}{|c|}{ Somatization } \\
\hline Positive & $26(12.56)$ & $24(42.86)$ & $2(1.32)$ \\
\hline Negative & $181(87.44)$ & $32(57.14)$ & $149(98.68)$ \\
\hline \multicolumn{4}{|c|}{ Obsessive-compulsive } \\
\hline Positive & $53(25.60)$ & $49(87.50)$ & $4(2.65)$ \\
\hline Negative & $154(74.40)$ & $7(12.50)$ & $147(97.35)$ \\
\hline \multicolumn{4}{|c|}{ Interpersonal sensitivity } \\
\hline Positive & $34(16.43)$ & $31(55.36)$ & $3(1.99)$ \\
\hline Negative & $173(83.57)$ & $25(44.64)$ & $148(98.01)$ \\
\hline \multicolumn{4}{|l|}{ Depression } \\
\hline Positive & $30(14.49)$ & $30(53.57)$ & $0(0.00)$ \\
\hline Negative & $177(85.51)$ & $26(46.43)$ & $151(100.00)$ \\
\hline \multicolumn{4}{|l|}{ Anxiety } \\
\hline Positive & $30(14.49)$ & $30(53.57)$ & $0(0.00)$ \\
\hline Negative & $177(85.51)$ & $26(46.43)$ & $151(100.00)$ \\
\hline \multicolumn{4}{|l|}{ Hostility } \\
\hline Positive & $31(14.98)$ & $30(53.57)$ & $1(0.66)$ \\
\hline Negative & $176(85.02)$ & $26(46.43)$ & $150(99.34)$ \\
\hline \multicolumn{4}{|c|}{ Photic anxiety } \\
\hline Positive & $20(9.66)$ & $20(35.71)$ & $0(0.00)$ \\
\hline Negative & $187(90.34)$ & $36(64.29)$ & $151(100.00)$ \\
\hline \multicolumn{4}{|c|}{ Paranoididefition } \\
\hline Positive & 25 (12.08) & $25(44.64)$ & $0(0.00)$ \\
\hline Negative & $182(87.92)$ & $31(55.36)$ & $151(100.00)$ \\
\hline \multicolumn{4}{|c|}{ Psychotieism } \\
\hline Positive & $25(12.08)$ & 25 (44.64) & $0(0.00)$ \\
\hline Negative & $182(87.92)$ & $31(55.36)$ & $151(100.00)$ \\
\hline \multicolumn{4}{|l|}{ Other } \\
\hline Positive & $56(27.05)$ & $38(67.86)$ & $18(11.92)$ \\
\hline Negative & $151(72.95)$ & $18(32.14)$ & $133(88.08)$ \\
\hline
\end{tabular}

and more night shifts may lead to insomnia, which was found in our study. Furthermore, more contact with patients with higher-severity illness was demonstrated to result in higher IES scores [23]. Nurses often have more contact with patients than doctors, which resulted in poor sleep quality of nurses [2]. Consistent with our finding, Jianbo Lai et al. [6] found medical workers in Wuhan showed more severe symptoms of insomnia compared with those outside Wuhan. These findings suggested more stress among medical workers in Wuhan, the epicenter of the pandemic in China, and their sleep quality might require special attention.

In addition, previous studies reported that females were more susceptible to insomnia $[11,24]$. However, in some studies, males were reported to be prone to poor sleep quality $[25,26]$. In the current study, we found that the sleep quality of males was worse than that of females, as well. The reasons might be that the percentage of male supporting
Wuhan $(72 \%, 23$ out of 32) was larger than females (45\%, 78 out of 175) in the current study, and the medical workers supporting Wuhan were more likely to suffer from insomnia, which led to the sleep quality of males was worse than that of females. Of note, although we tried our best to control the confounding factors through multivariate logistic analysis, potential confounding factors might still exist. This study also found that people with working years $>15$ years were more likely to suffer from insomnia, which might be related to the decline of physiological function. Moreover, medical workers with long working years often act as department directors or head nurses, so they need to coordinate and manage the work of the department, and consider more things, leading to the decline of sleep quality. Consistent with the study by Haitham Jahram et al. [11], there was no statistical correlation between front-line medical staff and insomnia after multivariate logistic analysis in the present study, but we found that front-line medical staffs were more prone to insomnia in univariate logistic analysis, suggesting that more attention should also be paid to the sleep problems of front-line medical staff [2].

As sleep and mental problems are complex multifactorial problems, the effect of a single factor may be weak, so we should focus on the interactions of multiple factors. However, due to the influence of "dimension disaster", the traditional statistical models are not suitable for exploring potential high-order interactions. Generalized multivariate dimension reduction (GMDR), as a non-parametric testing method, can overcome the influence of dimension and correct the confounding factors, which significantly improves the accuracy of prediction. With this method, a three-factor interaction among red meat intake, pickled vegetable, and cured meat intake was reported to increase the risk of colorectal cancer [27]. In this study, we found that there was a two-factor interaction of sleep quality among working years and working shifts, which means that more attention should be paid on the subjects with more night shifts and working years longer than 15 years. However, it is different between statistical interaction and biological interaction [18], whether these statistical interactions obtained in the current study have biological effects, and the specific mechanisms are still unclear, which should be explored in future research.

Owing to the sudden outbreak of the pandemic, strong infectivity, and the occurrence of multiple clinical medical staff infection, medical workers are susceptible to psychological burden. A cross-sectional study reported that the prevalence of psychological abnormality was $14.5 \%$ in medical workers during the COVID-19 [17]. Similarly, our study also showed the mental abnormality of medical workers, and the prevalence of mental abnormality was $27.05 \%$, mainly manifested as obsessive-compulsive symptom, indicating that the mental status of medical staff during the COVID-19 
Table 5 Logistic regression analysis of risk factors associated with mental health

\begin{tabular}{|c|c|c|c|c|c|}
\hline \multirow[t]{2}{*}{ Variables } & \multirow{2}{*}{$\begin{array}{l}\text { No. of cases/no. } \\
\text { of total cases (\%) }\end{array}$} & \multicolumn{2}{|l|}{ Model 1} & \multicolumn{2}{|l|}{ Model 2} \\
\hline & & OR (95\% CI) & $P$ & OR $(95 \% \mathrm{CI})$ & $P$ \\
\hline \multicolumn{6}{|l|}{ Age (years) } \\
\hline$\leq 30$ & $21 / 76(27.63)$ & 1.00 (reference) & NA & 1.00 (reference) & NA \\
\hline$>30$ & $35 / 131(26.72)$ & $0.96(0.51-1.80)$ & 0.89 & $1.88(0.66-5.37)$ & 0.24 \\
\hline \multicolumn{6}{|l|}{ Gender } \\
\hline Male & $10 / 32(31.25)$ & $1.28(0.56-2.89)$ & 0.56 & $3.22(0.96-10.79)$ & 0.06 \\
\hline Female & $46 / 175(26.29)$ & 1.00 (reference) & NA & 1.00 (reference) & NA \\
\hline \multicolumn{6}{|l|}{ Working years (years) } \\
\hline $1-5$ & $13 / 43(30.23)$ & 1.00 (reference) & NA & 1.00 (reference) & NA \\
\hline $6-10$ & 25/85 (29.41) & $0.96(0.43-2.14)$ & 0.92 & $2.62(0.62-11.17)$ & 0.19 \\
\hline $11-15$ & 9/39 (23.08) & $0.69(0.26-1.86)$ & 0.47 & $1.82(0.67-4.94)$ & 0.24 \\
\hline$>15$ & $9 / 40(22.50)$ & $0.67(0.25-1.80)$ & 043 & $1.43(0.46-4.42)$ & 0.53 \\
\hline \multicolumn{6}{|l|}{ Educational level } \\
\hline College & $11 / 29(37.93)$ & 1.00 (reference) & NA & 1.00 (reference) & NA \\
\hline Undergraduate & $43 / 166(25.90)$ & $0.57(0.25-1.31)$ & 0.19 & $0.71(0.28-1.79)$ & 0.47 \\
\hline Postgraduate & $2 / 12(16.67)$ & $0.33(0.06-1.78)$ & 0.20 & $0.45(0.06-3.16)$ & 0.42 \\
\hline \multicolumn{6}{|l|}{ Occupation } \\
\hline Doctor & $9 / 38(23.68)$ & 1.00 (reference) & NA & 1.00 (reference) & NA \\
\hline Nurse & 43/155 (27.74) & $1.24(0.54-2.83)$ & 0.61 & $2.18(0.62-7.65)$ & 0.22 \\
\hline Technician & 4/14 (28.57) & $1.29(0.32-5.12)$ & 0.72 & $0.76(0.14-4.23)$ & 0.75 \\
\hline \multicolumn{6}{|l|}{ Type of hospital } \\
\hline Grade I hospital or community & $2 / 8(25.00)$ & 1.00 (reference) & NA & 1.00 (reference) & NA \\
\hline Grade II hospital & $13 / 32(40.63)$ & $1.02(0.20-5.27)$ & 1.00 & $3.71(0.54-25.57)$ & 0.18 \\
\hline Grade III hospital & $41 / 167(24.55)$ & $2.10(0.96-4.63)$ & 0.07 & $1.39(0.24-8.10)$ & 0.72 \\
\hline \multicolumn{6}{|l|}{ Working shifts } \\
\hline As usual & $13 / 60(21.67)$ & 1.00 (reference) & NA & 1.00 (reference) & NA \\
\hline More night shifts & $18 / 60(30.00)$ & $1.54(0.68-3.54)$ & 0.30 & $2.05(0.83-5.04)$ & 0.12 \\
\hline More day shifts & 25/87 (28.74) & $1.46(0.68-3.15)$ & 0.34 & $1.68(0.72-3.94)$ & 0.23 \\
\hline \multicolumn{6}{|l|}{ Working position } \\
\hline Frontline & $32 / 132(24.24)$ & $0.68(0.36-1.27)$ & 0.23 & $0.87(0.32-2.40)$ & 0.79 \\
\hline Second line & $24 / 75(32.00)$ & 1.00 (reference) & NA & 1.00 (reference) & NA \\
\hline \multicolumn{6}{|l|}{ Supporting Wuhan } \\
\hline Yes & 22/101 (21.78) & $0.59(0.32-1.10)$ & 0.10 & $0.53(0.20-1.40)$ & 0.20 \\
\hline No & $34 / 106(32.08)$ & 1.00 (reference) & NA & 1.00 (reference) & NA \\
\hline
\end{tabular}

Model 1: univariate logistic analysis; model 2: multivariate logistic analysis pandemic was poor. However, no significant factors were found associated with mental symptom by logistic analysis and no interactions were found by GMDR analysis, which should be explored in future research.

Our study assessed the sleep quality and mental health status among medical workers during the COVID-19 pandemic, and explored associated factors and their interactions, which could help to provide precise interventions of sleep and mental problems for medical workers. However, there were several limitations. First, the causal association between demographic data and sleep quality or mental health status was not certain because of the cross-sectional design. Second, owing to the severe pandemic situation, no large scale was carried out, only the subjects in Ningbo were investigated, and the sample size was limited. Third, due to the time limitation of the pandemic, we conducted a rapid survey based on the Wenjuanxing program, no long-term survey was carried out, which might lead to a potential risk of bias in the way the data collected. Therefore, a prospective study with a large sample size is expected to be conducted, and more objective data on sleep quality and mental health status should be collected.

\section{Conclusion}

The findings indicated that about one-third of the medical workers suffered from sleep and mental problems during the COVID-19 pandemic. Sleep-related factors included gender, 
working years, occupation, working shifts, whether to support Wuhan, and a two-factor interaction between working years and working shifts. Interventions for sleep and mental problems among medical workers are needed.

Acknowledgements This work was supported by the General Research Project of Zhejiang Provincial Department of Education (Y202043652). We want to thank all medical workers, especially the frontline medical workers, for their cooperation and support.

\section{Compliance with ethical standards}

Conflict of interest The authors declare no competing interests.

Ethical approval The study design was approved by the ethics committee of Ningbo Medical Center Lihuili Hospital.

Informed consent Informed consent was obtained from all individual participants included in the study.

\section{References}

1. World Health Organization. Coronavirus disease 2019. https:// www.who.int/emergencies/diseases/novel-coronavirus-2019. Accessed 2 Sep 2020.

2. Zhang C, Yang L, Liu S, Ma S, Wang Y, Cai Z, et al. Survey of insomnia and related social psychological factors among medical staff involved in the 2019 novel coronavirus disease outbreak. Front Psychiatry. 2020;11:306.

3. Wu K, Wei X. Analysis of psychological and sleep status and exercise rehabilitation of front-line clinical staff in the fight against covid-19 in china. Med Sci Monit Basic Res. 2020;26:e924085.

4. Tsamakis K, Rizos E, Manolis AJ, Chaidou S, Kympouropoulos S, Spartalis E, et al. Covid-19 pandemic and its impact on mental health of healthcare professionals. Exp Ther Med. 2020;19(6):3451-3.

5. Lu W, Wang H, Lin Y, Li L. Psychological status of medical workforce during the covid-19 pandemic: a cross-sectional study. Psychiatry Res. 2020;288:112936.

6. Lai J, Ma S, Wang Y, Cai Z, Hu J, Wei N, et al. Factors associated with mental health outcomes among health care workers exposed to coronavirus disease 2019. JAMA Netw Open. 2020;3(3):e203976.

7. Kang L, Ma S, Chen M, Yang J, Wang Y, Li R, et al. Impact on mental health and perceptions of psychological care among medical and nursing staff in Wuhan during the 2019 novel coronavirus disease outbreak: a cross-sectional study. Brain Behav Immun. 2020;87:11-7.

8. Xing J, Sun N, Xu J, Geng S, Li Y. Study of the mental health status of medical personnel dealing with new coronavirus pneumonia. PLoS ONE. 2020;15(5):e0233145.

9. Bai Y, Lin CC, Lin CY, Chen JY, Chue CM, Chou P. Survey of stress reactions among health care workers involved with the sars outbreak. Psychiatr Serv. 2004;55(9):1055-7.

10. Lee AM, Wong JG, McAlonan GM, Cheung V, Cheung C, Sham PC, et al. Stress and psychological distress among sars survivors 1 year after the outbreak. Can J Psychiatry. 2007;52(4):233-40.

11. Jahrami H, BaHammam AS, AlGahtani H, Ebrahim A, Faris $\mathrm{M}$, AlEid $\mathrm{K}$, et al. The examination of sleep quality for frontline healthcare workers during the outbreak of covid-19. Sleep Breath. 2020;24(2):1-9.

12. Buysse DJ, Reynolds CF 3rd, Monk TH, Hoch CC, Yeager AL, Kupfer DJ. Quantification of subjective sleep quality in healthy elderly men and women using the Pittsburgh sleep quality index (PSQI). Sleep. 1991;14(4):331-8.

13. Tsai PS, Wang SY, Wang MY, Su CT, Yang TT, Huang CJ, et al. Psychometric evaluation of the Chinese version of the pittsburgh sleep quality index (CPSQI) in primary insomnia and control subjects. Qual Life Res. 2005;14(8):1943-52.

14. Xiao H, Zhang Y, Kong D, Li S, Yang N. The effects of social support on sleep quality of medical staff treating patients with coronavirus disease 2019 (covid-19) in January and February 2020 in China. Med Sci Monit. 2020;26:e923549.

15. Derogatis LR, Lipman RS, Covi L. Scl-90: an outpatient psychiatric rating scale-preliminary report. Psychopharmacol Bull. 1973;9(1):13-28.

16. Derogatis LR, Unger R. Symptom checklist-90-revised. Corsini Encycl Psychol. 2010;1:1-2.

17. Cai W, Lian B, Song X, Hou T, Deng G, Li H. A cross-sectional study on mental health among health care workers during the outbreak of corona virus disease 2019. Asian J Psychiatr. 2020;51:102111.

18. Lou XY, Chen GB, Yan L, Ma JZ, Zhu J, Elston RC, et al. A generalized combinatorial approach for detecting gene-by-gene and gene-by-environment interactions with application to nicotine dependence. Am J Hum Genet. 2007;80(6):1125-37.

19. Chen Q, Tang X, Hu YH. detecting interaction for quantitative trait by generalized multifactor dimensionality reduction. Zhonghua Liu Xing Bing Xue Za Zhi. 2010;31(8):938-41.

20. Lee S, Chan LY, Chau AM, Kwok KP, Kleinman A. The experience of sars-related stigma at amoy gardens. Soc Sci Med. 2005;61(9):2038-46.

21. Su TP, Lien TC, Yang CY, Su YL, Wang JH, Tsai SL, et al. Prevalence of psychiatric morbidity and psychological adaptation of the nurses in a structured sars caring unit during outbreak: a prospective and periodic assessment study in Taiwan. J Psychiatr Res. 2007;41(1-2):119-30.

22. Jehan S, Zizi F, Pandi-Perumal SR, Myers AK, Auguste E, JeanLouis G, et al. Shift work and sleep: medical implications and management. Sleep Med Disord. 2017;1(2):1-14.

23. Maunder RG, Lancee WJ, Rourke S, Hunter JJ, Goldbloom D, Balderson K, et al. Factors associated with the psychological impact of severe acute respiratory syndrome on nurses and other hospital workers in toronto. Psychosom Med. 2004;66(6):938-42.

24. Zhang B, Wing YK. Sex differences in insomnia: a meta-analysis. Sleep. 2006;29(1):85-93.

25. Cheng SH, Shih CC, Lee IH, Hou YW, Chen KC, Chen KT, et al. A study on the sleep quality of incoming university students. Psychiatry Res. 2012;197(3):270-4.

26. Mamun MA, Hossain MS, Kamruzzaman M, Khalil MI, Sikder MT, Manzar MD, et al. Prevalence of poor sleep quality and its determinants among bangladeshi students: a pilot study. Sleep Vigil. 2020;9:1-9.

27. Li YL, Feng F, Yan J, Chen LL, Li XL, Liu WH, et al. association between cured meat consumption and risk of colorectal cancer in people with different dietary habits and lifestyles. Zhonghua Liu Xing Bing Xue Za Zhi. 2016;37(7):1006-11.

Publisher's Note Springer Nature remains neutral with regard to jurisdictional claims in published maps and institutional affiliations. 